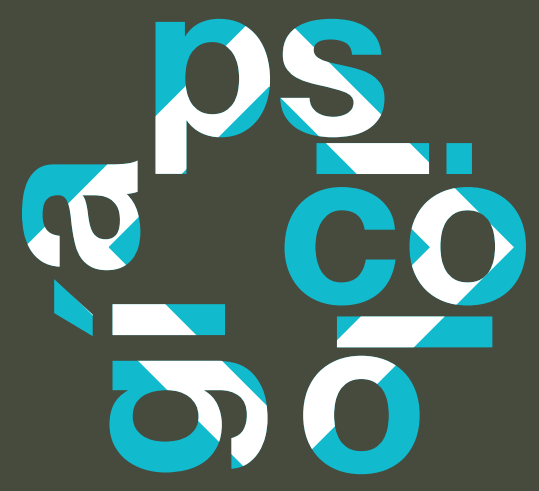

\title{
Anuario de
}

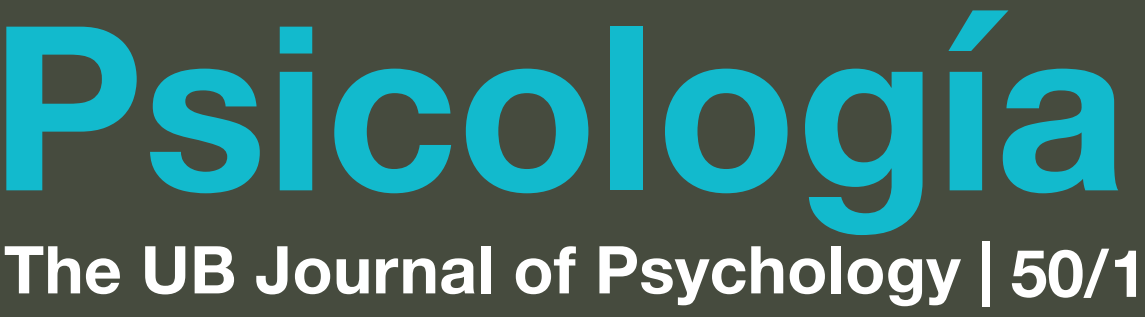

Raquel Suriá Martínez

raquel.suria@ua.es

Departamento de Comunicación

y Psicología Social

Edificio de Ciencias Sociales

Universidad de Alicante

Carretera San Vicente del Raspeig, s/n

03690 San Vicente del Raspeig (Alicante)

Anuario de Psicología

N. ${ }^{\circ}$ 50/I | 2020 | págs. 29-37

Recibido: 9 de julio de 2018

Aceptado: 22 de enero de 2020

DOI: IO.1344/ANPSIC2020.50.2

ISSN: 0066-5 I 26 | (C) 2020 Universitat de Barcelona. All rights reserved.
Empoderamiento

y rendimiento académico

en estudiantes de educación secundaria obligatoria con y sin discapacidad

\section{Raquel Suriá Martínez Esther Villegas Castrillo}

\section{Resumen}

Introducción. Conocer cómo afecta el empoderamiento en el rendimiento académico en los estudiantes con discapacidad es uno de los puntos de partida para facilitar la elaboración de elementos instruccionales ajustados para este colectivo. El objetivo de este trabajo consistió en conocer el nivel de empoderamiento y su influencia en el rendimiento académico entre los estudiantes universitarios en función de si tienen discapacidad o no. Asimismo, se analizó según el género y la edad de los estudiantes.

Método. Participaron 693 estudiantes de Educación Secundaria Obligatoria. De ellos, 67 tenían discapacidad y 626 no tenían ninguna discapacidad. Se utilizó el cuestionario elaborado por Rogers, Chamberlin, Ellison, y Crean (1997), diseñado para medir el nivel de empoderamiento conformado por 5 factores (Autoestima-Autoeficacia, Poder/Impotencia, Autonomía, Optimismo-Control sobre el futuro, Ira apropiada). El cuestionario está formado por un total de 28 ítems, con respuesta tipo Likert de 4 puntos ( 0 = nada de acuerdo a 3 = muy de acuerdo).

Resultados. Los análisis indicaron un nivel menos elevado de empoderamiento entre los estudiantes con discapacidad, especialmente en chicas con menos edad. Asimismo, el nivel de rendimiento académico fue inferior en los estudiantes más jóvenes con discapacidad. No se observaron diferencias estadísticamente significativas en el rendimiento académico en función del sexo. Finalmente, se encontró una relación positiva entre el rendimiento académico y el empoderamiento. Del mismo modo, se encontró una relación positiva entre el rendimiento académico y los jóvenes estudiantes sin discapacidad.

Discusión. Los resultados reflejan la importancia de potenciar el empoderamiento en los jóvenes estudiantes con discapacidad para que dispongan de las habilidades necesarias para un adecuado ajuste y éxito académico.

\section{Pallabras clave}

Estudiantes, universidad, discapacidad, empoderamiento, potenciación, rendimiento académico 


\title{
Empowerment and Academic Performance in Compulsory Secondary Education Students with and without Disability
}

\begin{abstract}
Introduction: Knowledge about the influence of empowerment on academic performance in students with disabilities is essential to the educational success of this group. The objective of this study was to analyze the level of empowerment and its influence on academic performance in university students based on whether they have disabilities or not. An analysis by gender and age was also carried out.
\end{abstract}

Method: Of the 693 students who participated, 67 had some disability and 626 had no disability. The study used the questionnaire drawn up by Rogers, Chamberlin, Ellison and Crean (1997), designed to measure the level of empowerment using 5 factors (Self-esteem / Self-efficacy; Power / Impotence; Autonomy; Optimism / Control over the future; Appropriate anger). The questionnaire is made up of 28 items with Likert response items rated from 0 to 3 ( $0=$ strongly disagree, to $3=$ strongly agree).

Results: The analysis shows less empowerment among young people with disabilities, especially in younger students and girls. Likewise, the level of academic performance was lower in younger students with disabilities. There were no statistically significant differences in academic performance according to sex. Finally, a positive relationship between academic achievement and empowerment was found. A positive relationship was also found between academic performance and young students without disabilities.

Discussion. The results show the importance of enhancing empowerment in young students with disabilities to ensure that they have the skills needed for an appropriate adjustment and academic success.

\section{Keywords}

Students; university; disability; empowerment; enhancement; academic performance

\section{INTRODUCCIÓN}

Uno de los contextos más importantes desde el que se está fomentando la integración de los jóvenes con discapacidad es el ámbito educativo (Polo-Sánchez y López-Justicia, 2012; Rodríguez-Martín y Álvarez-Arregui, 2015; Suriá, Villegas, y Rosser Limiñana, 2017). Así, son muchos los factores que se deben tener en cuenta desde cualquier política educativa, que van desde la supresión de barreras arquitectónicas hasta las adaptaciones curriculares (Alcantud, Ávila-Clemente, y Asensi-Borrás, 2000). Por tal motivo, son numerosos los estudios en el marco internacional que han analizado las experiencias de los estudiantes con discapacidad y las repercusiones que estas tienen en el ámbito personal y académico (Figuera-Gazo y Coiduras-Rodríguez, 2013; Peralta, Gandoy, Jara, y Pacenza, 2016; Suriá, 20184).

Entre las ivestigaciones más relevantes que abordan la problemática académica de los estudiantes con discapacin dad, destacata la de Borland y James (1999) sobre las expe- riencias sociales y el aprendizaje de más de 7000 alumnos en Gran Bretaña y sobre los mecanismos de aseguramiento de la calidad para el alumnado con este hándicap. Sus conclusiones fueron replicadas en otras investigaciones más recientes, coincidiendo todas en destacar las dificultades que una gran parte de los estudiantes encuentra para afrontar esta etapa formativa y que afectan, en la mayoría de los casos, a las metas académicas de los estudiantes (De los Ríos Gutiérrez y González, 2016; Figuera-Gazo y Coiduras, 2013; Matalinares et al., 2013; Suriá, 2018).

En este sentido, es aceptado entre el contexto académico analizar el éxito académico a través del rendimiento académico, definido como la suma de diferentes y complejos factores que interactúan en el aprendizaje de la persona, siendo un valor atribuido al logro del estudiante en las tareas académicas y, por tanto, imprescindible para la consecución de los objetivos o metas que hay que alcanzar (Mohedano y Guadalupe, 2006). Así, el rendimiento 
académico puede considerarse como un indicador, no únicamente como una forma de medir el éxito de los estudiantes en el ámbito académico, sino también de la calidad de una institución, más adecuadamente, de la cam lidad de la enseńanza en una determinada etapa. De este modo, y siguiendo a Fullan y Escudero (2002, p. 254), las calificaciones son una medida de los resultados de la enseñanza, no solo por la calidad de los alumnos, sino también por el criterio y el rigor personal del profesor a la hora de diseñar la enseñanza y de valorar y calificar el aprendizaje y el rendimiento académico.

$\mathrm{Si}$ se atiende a los factores influyentes en el rendimiento académico de los estudiantes con discapacidad, además de la multitud de variables del entorno (barreras arquitectónicas, actitudinales, etc.) y de los aspectos relacionados con la propia discapacidad de cada estudiante (tipología, grado de severidad, etc.), existen multitud de factores personales que contribuyen a explicar las experiencias de transición de los estudiantes con discapacidad en positivo, como éxito, o en negativo, como fracaso, y que abarcarían desde las variables estructurales o familiares (Gómez-Puerta y Cardona, 2010; Suriá, 2018), como los factores sociodemográficos, la edad (Sepúlveda-Rueda, 2008) y el sexo (Botero, 2013), hasta, incluso, otras variables de índole psicológico (Matalinares et al., 2013).

En referencia a este último tipo de variables, desde la psicología positiva se enfatiza en el estudio de los factores psicológicos protectores. Así, por ejemplo, diferentes autores (Figuera-Gazo et al., 2013; González y Roses, 2016; Wehmeyer, 2009) confirman la incidencia de las variables psicológicas y concluyen que los estudiantes con discapacidad con resultados positivos presentan mayor capacidad para fijar objetivos y perseverar en sus logros (Elizur y Magyari, 1993), y una tendencia a implicarse en acciones posibles y a comunicar sus intereses, preferencias y necesidades (González y Roses, 2016). Todas estas variables están relacionadas con lo que se conoce hoy como empoderamiento.

Este constructo surgió con un planteamiento de carácter psicosocial hacia la discapacidad, de la mano de diferentes autores (Barnes, Mercer, y Shakespeare, 1997; Comité Español de Representantes de Personas con Discapacidad (CERMI), 2017; Moore, Beazley, y Maelzer, 1998; Oliver, 1998; por citar algunos de los más representativos), con la finalidad de dar un giro a los enfoques biomédicos, y hace referencia a la capacidad psicológica de cada persona para el desarrollo de un locus de control interno, autoeficacia y habilidades para alcanzar metas y objetivos definidos por ella misma, abordando de este modo la vida en términos de oportunidades personales y sociales (Rappaport, 1984; Segado, 2011; Zimmerman, 2000).

En el contexto académico, diferentes autores (González y Roses, 2016; Matalinares et al., 2013; Peralta et al., 2016; Silva, Kronmüller, Cruz, y Riffo, 2015; Suriá, Castrillo, y Rosser Limiñana, 2017; Zimmerman, 2000) han encontrado que el empoderamiento podría actuar como un potenciador en el logro de un desempeño satisfactorio de los estudiantes, ya que contribuiría a un afrontamiento de los estresores propios de su ambiente, con mayores probabilidades de éxito.

Así, aunque es amplia la literatura existente centrada en el análisis de las variables implicadas en la transición a la vida adulta en los jóvenes con discapacidad (De los Ríos Gutiérrez y González, 2016; Gómez-Puerta y Cardona, 2010; Matalinares et al., 2013; Polo-Sánchez y López-Justicia, 2012; Rodríguez-Martín y Álvarez-Arregui, 2015; Suriá et al., 2018), no es tan extensa la dedicada a profundizar en la relación del empoderamiento y el rendimiento académico de los estudiantes con discapacidad (Peralta et al., 2016; Silva et al., 2015; Suriá et al., 2017; Suriá, 2016).

Analizar la relación entre este constructo y el rendimiento académico en una población con discapacidad cobra especial relevancia, puesto que, tal y como indica Wehmeyer (2009), el empoderamiento es el proceso por el cual la acción de una persona es el principal agente causal de su propia vida, así como de las elecciones sobre la calidad de esta, de tal forma que esta autodeterminación ayudaría, y debería ser contemplada desde los servicios pedagógicos, a propiciar que estos estudiantes sean los auténticos protagonistas en la toma de decisiones sobre su vida académica (Matalinares et al., 2013).

Por tanto, y a partir de la importancia de contribuir en el diseño de políticas educativas que procuren el desarrollo de constructos para el éxito de la formación del alumnado, el objetivo de este trabajo es conocer el nivel de empoderamiento y su influencia en el rendimiento académico entre los jóvenes estudiantes con discapacidad. Este objetivo general se desglosa en los siguientes objetivos específicos:

1. Conocer el nivel de empoderamiento de los estudiantes de secundaria. Esto se analiza en función de tener o no discapacidad, de la edad y del sexo.

2. Identificar si existen diferencias en el rendimiento académico de los estudiantes. Asimismo se analiza en función de tener o no discapacidad, de la edad y del sexo.

3. Analizar si el nivel de empoderamiento, el tener o no discapacidad, la edad y el sexo pueden influir directamente en el rendimiento académico de los estudiantes.

\section{MÉTODO}

\section{Participantes}

Se ha estudiado la serie de casos transversal constituida por una muestra intencional, por motivos de accesibilidad, de estudiantes con discapacidad. La muestra está formada por 693 estudiantes que están finalizando sus estudios en institutos de la ciudad de Alicante. De ellos, 
el $76.6 \%$ eran mujeres y un $23.4 \%$, varones. En referencia al rango de edad, se encontraba entre los 15 y 18 años, siendo el más frecuente el de 15 a 17 (52.7\%). Con respecto a la discapacidad, 67 de ellos tenían discapacidad y 626 no tenína ninguna discapacidad. La distribución según el tipo de discapacidad que tenían fue: un $25.8 \%$, discapacidad intelectual; un $20.0 \%$, discapacidad motora; un $17.4 \%$, pluridiscapacidad, un $17.4 \%$, discapacidad visual, un $15.8 \%$, discapacidad auditiva, y un $4.7 \%$ no la indican.

\section{Procedimiento}

La primera fase fue mantener una serie de reuniones preliminares con los responsables de la Dirección Territorial de la Conselleria de Educación, Cultura y Deporte de Alicante para estudiar la viabilidad del proyecto y los pasos que se tenían que seguir para contar con las autorizaciones pertinentes. Posteriormente, se solicitó autorización a la Secretaría Autonómica de Educación y Formación de la Conselleria de Educación, Cultura y Deporte de la Comunidad Valenciana para la realización del proyecto de investigación-acción. A continuación, tras la coordinación con la dirección de los IIES, se solicitó el consentimiento informado por escrito de los padres autorizando a sus hijos a participar en la investigación. Los cuestionarios fueron contestados anónima y colectivamente en el aula. Se procedió a la entrega de los ejemplares con las instrucciones y de la hoja de respuesta. Seguidamente, se leyeron en voz alta las instrucciones y se enfatizó en la importancia de no dejar ninguna pregunta sin contestar. Los investigadores estuvieron presentes durante la administración de las pruebas para aclarar las posibles dudas. El tiempo estimado de aplicación fue de aproximadamente quince minutos.

\section{Instrumentos}

El cuestionario constaba de dos partes. En la primera se incluían preguntas para obtener el perfil sociodemográfico de los participantes (sexo, edad, nivel de funcionalidad y antigüedad de la lesión). La segunda era la escala elaborada por Rogers, Chamberlin, Ellison, y Crean (1997), diseñada para medir el nivel de empoderamiento. Se empleó la versión traducida al castellano por Suriá (2014), destinada a examinar el empoderamiento de los progenitores de hijos con discapacidad. La escala está formada por un total de 28 ítems, con respuesta tipo Likert de 4 puntos ( 1 = nada de acuerdo, 4 = muy de acuerdo). La información solicitada recoge aspectos en relación a la percepción del propio sujeto en función de la capacidad para la toma de decisiones. La puntuación máxima es de 84 puntos, estableciéndose la medida de corte en tres rangos (nivel bajo $=$ de 0 a 27 , nivel medio $=$ de 28 a 56 y nivel alto $=$ de 57 a 84 ).

Se eligió esta escala por su fácil aplicación, por estar validada para población joven y adulta y, finalmente, por las propiedades psicométricas que muestra la versión original (Rogers et al., 1997). Así, se ha indicado una fiabilidad adecuada $(\mathrm{a}=.86)$ y una validez que mostró una varianza explicada del $53.9 \%$, definida por cinco factores: Autoestima-Autoeficacia (explica el $24.5 \%$ de la varianza total); Poder/Impotencia (explica el $12.4 \%$ de la varianza total); Activismo comunitario-Autonomía (explica el $7.6 \%$ de la varianza total); Optimismo-Control sobre el futuro (explica el $5.4 \%$ de la varianza total), e Ira apropiada (explica el $4 \%$ de la varianza total). Asimismo, la versión traducida (Suriá et al., 2017) mostró una fiabilidad del $88 \%$ y una varianza que explica el $72.92 \%$ de la variabilidad. Para comprobar si la escala se adecuaba a las características de este estudio, se comprobaron las propiedades psicométricas de la escala en la muestra. Para ello, se utilizó el Análisis Factorial con Componentes Principales (AFECP), que mostraba una varianza explicada del $50.82 \%$, distribuida en 5 factores. Previamente el test de esfericidad de Barlett $\left[\chi^{2}=111.576(\mathrm{p}<.001)\right]$ y la medida de adecuación muestral KMO (0.582) indicaron ser favorables para realizar el AFECP. La fiabilidad del cuestionario fue adecuada $((\mathrm{a}=.84)$.

\section{Rendimiento académico}

Este se determinó mediante el promedio, con una valoración cuantitativa de las calificaciones académicas acumuladas desde la entrada en el instituto hasta el momento de la cumplimentación del cuestionario. Se utilizó este criten rio según las recomendaciones de Allen (2002), que indica que las calificaciones académicas representan la manera más práctica y más comúnmente utilizada para evaluar el grado de cumplimiento de los objetivos de aprendizaje y el grado de éxito académico dentro del ciclo formativo. De este modo, se solicitó en uno de los ítems incluido al final del Cuestionario sociodemográfico que los alumnos se puntuasen en una escala del 1 a 10.

\section{Análisis estadístico}

Para examinar los datos sociodemográficos hallamos las frecuencias y los porcentajes. Para comprobar si existen diferencias estadísticamente significativas en el grado de empoderamiento y en el rendimiento académico en función de tener o no discapacidad y en función del sexo se utilizó la $t$ de Student para dos muestras independientes. Para las comparaciones de más de dos grupos (edad) se utilizó el ANOVA. Previo a esto, se comprobó que se cumplían los supuestos de homocedasticidad de varianza, normalidad de las distribuciones e independencia de variables. Esto se comprobó a través de la prueba de Levene, de Kolmogorov-Smirnov y Chi cuadrado respectivamente. Asimismo, se calculó el tamaño del efecto (diferencia media tipificada o índice $d$; Cohen, 1988), el cual indica si la $o$ magnitud de las diferencias halladas es pequeña, moderada o grande. 
Finalmente, con el objetivo de deslindar la asociación del rendimiento académico con las variables independientes posiblemente relacionadas (tener discapacidad o no, sexo, edad y empoderamiento), se calculó el coeficiente de correlación de Pearson y se aplicó un modelo de regresión lineal múltiple. Para cumplir los requisitos del modelo, los datos se transformaron según el modelo de transformaciones potenciales desarrollado por Box y Cox (1982). Se utilizó esta prueba, puesto que permite estimar la transformación potencial más adecuada cuando no hay una razón a priori para elegir un modelo concreto de transformación.

\section{RESULTADOS}

Con respecto al primer objetivo: examinar las medias de empoderamiento de los estudiantes, en primer lugar, se observó que los estudiantes reflejaron un nivel de empoderamiento adecuado $(M=56.86, D T=10.14)$. Al examinar si difiere el empoderamiento en función de las variables analizadas en el estudio, esto es, tener o no discapacidad, la edad y el sexo, se observaron los siguientes resultados (tabla 1):
En lo relativo al nivel de empoderamiento en función de tener discapacidad o no tenerla se observa un índice más elevado en los estudiantes sin discapacidad, $\left[t_{(691)}=9.89\right.$, $p<.001]$, observándose un tamaño del efecto elevado $(d=1.43)$. En referencia a cada uno de los factores en función de esta variable, se observaron diferencias estadísticamente significativas en los factores relacionados con la Autoestima, factor $1,\left[t_{(691)}=6.79, p<.001(d=0.88)\right]$, con el Poder/Impotencia, factor $2\left[t_{(69) 1)}=7.08, p<.001\right.$ $(d=0.70)]$, el factor referente a la Autonomía, factor 3, $\left[t_{(691)}=8.19, p<.001(d=0.76)\right]$ y el factor 4 , Optimismo-Control de futuro, $\left[t_{(691)}=2.04 ; p<.05(d=0.61)\right]$, indicando los jóvenes sin discapacidad medias más elevadas que los participantes con discapacidad.

En cuanto al nivel de empoderamiento en función de la edad se observaron diferencias estadísticamente significativas en la escala total, $\left[F_{(691)}=4.52, p<.05\right]$, de manera que el grupo de estudiantes de más edad fue el que indicó medias más elevadas que el resto de los grupos. Estos resultados se observaron en todos los factores de la escala. Los tamaños del efecto oscilan entre pequeños y moderados $(d=0.32-0.66)$. Del mismo modo se observaron diferencias estadísticamente significativas en el nivel de empoderamiento entre los estudiantes en función del sexo,

Tabla 1. Puntuaciones medias y desviaciones típicas en empoderamiento en función de tener o no discapacidad, de la edad y del sexo

\begin{tabular}{|c|c|c|c|c|c|c|c|c|c|c|c|c|}
\hline & \multicolumn{2}{|c|}{$\begin{array}{l}\text { Factor } 1 \\
\text { Autoestima- } \\
\text { Autoeficacia }\end{array}$} & \multicolumn{2}{|c|}{$\begin{array}{c}\text { Factor } 2 \\
\text { Poder/ } \\
\text { Impotencia }\end{array}$} & \multicolumn{2}{|c|}{$\begin{array}{c}\text { Factor } 3 \\
\text { Autonomía }\end{array}$} & \multicolumn{2}{|c|}{$\begin{array}{c}\text { Factor } 4 \\
\text { Optimismo- } \\
\text { Control futuro }\end{array}$} & \multicolumn{2}{|c|}{$\begin{array}{c}\text { Factor } 5 \\
\text { Ira apropiada }\end{array}$} & \multicolumn{2}{|c|}{$\begin{array}{c}\text { Escala } \\
\text { total }\end{array}$} \\
\hline & $\mathbf{M}$ & DT & $\mathbf{M}$ & DT & $\mathbf{M}$ & DT & $\mathbf{M}$ & DT & $\mathbf{M}$ & DT & $\mathbf{M}$ & DT \\
\hline Total & 14.68 & 4.17 & 14.43 & 2.55 & 10.12 & 3.18 & 3.84 & 1.29 & 3.37 & 1.09 & 56.86 & 10.14 \\
\hline \multicolumn{13}{|l|}{ Tener Disc. o no } \\
\hline Sin discapacidad & 18.22 & 3.77 & 14.98 & 3.05 & 13.71 & 2.76 & 4.22 & 1.09 & 3.70 & 1.43 & 57.91 & 7.01 \\
\hline Con discapacidad & 15.37 & 7.41 & 11.36 & 4.10 & 10.06 & 4.61 & 3.97 & 1.08 & 4.36 & 1.80 & 44.15 & 16.11 \\
\hline$t(p)$ & 6.79 & $(.001)$ & 7.08 & $(.000)$ & 8.19 & $(.000)$ & 2.04 & $(.049)$ & 2.27 & $(.020)$ & 9.89 & $(.000)$ \\
\hline \multicolumn{13}{|l|}{ Edad } \\
\hline $18-22$ & 13.07 & 4.16 & 14.46 & 1.54 & 9.74 & 3.38 & 3.39 & 1.27 & 2.92 & 0.99 & 53.78 & 10.31 \\
\hline $23-27$ & 17.76 & 4.57 & 14.84 & 3.08 & 10.01 & 3.27 & 4.09 & 1.45 & 3.48 & 1.09 & 57.46 & 11.87 \\
\hline $28-32$ & 17.79 & 3.47 & 13.96 & 3.75 & 10.37 & 2.89 & 4.58 & 0.94 & 3.60 & 0.78 & 57.98 & 6.78 \\
\hline $\mathbf{F}(\mathbf{p})$ & 1.17 & $(.037)$ & .73 & $(.045)$ & .24 & $(.046)$ & 1.89 & $(.006)$ & 2.97 & $(.046)$ & 4.52 & $(.040)$ \\
\hline \multicolumn{13}{|l|}{ Sexo } \\
\hline Varón & 17.99 & 3.54 & 14.32 & 1.92 & 12.11 & 2.24 & 3.86 & 1.28 & 3.30 & 1.91 & 54.61 & 10.01 \\
\hline Mujer & 16.73 & 4.08 & 13.94 & 2.06 & 10.74 & 2.18 & 3.79 & 1.14 & 3.29 & 1.82 & 54.40 & 9.86 \\
\hline $\mathbf{t}(\mathbf{p})$ & 4.07 & $(.042)$ & 2.37 & $(.243)$ & 3.78 & $(.045)$ & 2.60 & $(.288)$ & 1.81 & $(.082)$ & 3.89 & $(.098)$ \\
\hline
\end{tabular}


encontrándose puntuaciones más elevadas en los varones en el factor $1\left[t_{(691)}=4.07, p<0.5(d=0.34)\right]$ y en el factor $3\left[t_{(691)}=3.78, p<0,5(d=0.40)\right]$.

En cuanto al segundo objetivo: conocer el nivel de rendimiento académico, los resultados reflejan un nivel de rendimiento adecuado $(M=7.09, D T=0.82)$.

$\mathrm{Al}$ atender al nivel de rendimiento académico de los alumnos en función de tener o no discapacidad, se observa que los estudiantes con discapacidad $(M=7.02, D T=0.89)$ indican unas puntuaciones medias menores que los estudiantes $\sin$ discapacidad $(M=6.49, D T=1.11),\left[t_{(691)}=\right.$ $10.41, p<.05(d=0.42)]$. En relación con el rendimiento académico según la edad, fueron los participantes de más edad los que mostraron mejores resultados que el resto de los grupos, $\left[F_{(691)}=1.33, p<.05\right]$. Los tamaños del efecto fueron de magnitud moderada $(d=0.36-0.44)$. No se observó un efecto significativo en el rendimiento académico de los estudiantes en función del sexo $\left[t_{(691)}=2.04\right.$, $p=.058]$.

Tabla 2. Puntuaciones medias y desviaciones típicas en rendimiento académico en función de tener o no discapacidad, de la edad y del sexo

\begin{tabular}{|c|c|c|c|c|c|}
\hline & \multicolumn{3}{|c|}{ Rendimiento académico } & \multirow{2}{*}{\multicolumn{2}{|c|}{$\begin{array}{c}\text { Sig. } \\
\text { Estadística }\end{array}$}} \\
\hline & & $\mathbf{M}$ & DT & & \\
\hline \multirow{3}{*}{$\begin{array}{l}\text { Tener o no } \\
\text { discapacidad }\end{array}$} & Sin discapacidad & 8.91 & 0.91 & \multirow{2}{*}{10.41} & \multirow{2}{*}{.041} \\
\hline & Con discapacidad & 6.49 & 1.11 & & \\
\hline & $18-22$ & 7.11 & 0.97 & \multirow{3}{*}{1.33} & \multirow{3}{*}{.048} \\
\hline \multirow[t]{2}{*}{ Edad } & $23-27$ & 6.82 & 0.98 & & \\
\hline & $28-32$ & 7.87 & 1.01 & & \\
\hline \multirow{3}{*}{ Sexo } & Varón & 6.89 & 0.90 & \multirow{3}{*}{2.04} & \multirow{3}{*}{.058} \\
\hline & Mujer & 7.70 & 0.88 & & \\
\hline & Total & 7.02 & 0.89 & & \\
\hline
\end{tabular}

En referencia a los resultados del tercer objetivo: identificar si el nivel de empoderamiento puede influir directamente en el rendimiento académico de los estudiantes, el coeficiente de correlación indicó que el rendimiento académico se asoció de forma directa con el empoderamiento $(\mathrm{r}=.481, p<.000)$, así como con el hecho de no tener discapacidad $(\mathrm{r}=.363, p<.05)$. Al incluir las variables (tener o no discapacidad, sexo, edad y empoderamiento) en un modelo de análisis de regresión múltiple, se observó un valor de ANOVA estadísticamente significativo $\left[F_{(691)}=5.42, p<.05\right]$, con unos coeficientes estandarizados estadísticamente significativos $(p<.05)$ para la variable tener o no discapacidad y para el empoderamiento, observándose una relación positiva entre el empoderamiento y el rendimiento académico y entre este y los jóvenes estudiantes sin discapacidad (tabla 3).
Tabla 3. Coeficientes y niveles de significación para las variables incluidas en el modelo

\begin{tabular}{|c|c|c|c|c|c|}
\hline $\begin{array}{l}\text { Rendimiento } \\
\text { académico }\end{array}$ & $\mathbf{B}$ & $\begin{array}{l}\text { Error } \\
\text { típico }\end{array}$ & Beta & $t$ & $p$ \\
\hline Constante & 4.88 & .712 & & 8.006 & .001 \\
\hline $\begin{array}{l}\text { Tener o no } \\
\text { discapacidad }\end{array}$ & 3.569 & .076 & 3.058 & 2.876 & .031 \\
\hline Sexo & 2.604 & .082 & 2.154 & 1.52 & .061 \\
\hline Edad & 1.458 & 1.27 & .166 & 1.643 & .151 \\
\hline Empoderamiento & 3.012 & .108 & 2.02 & 4.156 & .035 \\
\hline $\begin{array}{l}\text { Variable } \\
\text { dependiente: } \\
\text { rendimiento } \\
\text { académico }\end{array}$ & & & & & \\
\hline
\end{tabular}

\section{DISCUSIÓN}

La necesidad de generar nuevos conocimientos sobre los jóvenes estudiantes que conviven con una discapacidad es el principal motor de este estudio. Así, es en el contexto educativo en el que el empoderamiento cobra relevancia a la hora de dotar a los estudiantes con este hándicap de recursos para enfrentarse exitosamente a los múltiples obstáculos con los que se enfrentan al cursar sus estudios.

Así, los resultados del primer objetivo, es decir, medir el grado de empoderamiento de estos jóvenes estudiantes, revelan que el nivel de empoderamiento en el alumnado se encuentra elevado, sin embargo, se observa que es inferior en los estudiantes con discapacidad. $\mathrm{Al}$ atender a los componentes de este, es decir, Autoestima-Autoeficacia, Poder/Impotencia, Autonomía, Optimismo-Control sobre el futuro e Ira apropiada, podemos entender que algunas de estas dimensiones se vean mermadas entre los jóvenes con discapacidad, los cuales, en alguna medida, tienen asociada una dependencia en alguno o varios ámbitos de su vida. Esto indica en cierto modo que, aunque no todos los estudiantes con discapacidad se encuentran en una situación de dependencia, sí que sucede en una gran mayoría de ellos. Esta dependencia puede afectar a cómo se ve uno mismo, no solo físicamente y desde una perspectiva académica/profesional y social, sino también personal, así como a la toma de decisiones y en general a su conducta (Alcantud et al., 2000; De los Ríos Gutiérrez y González, 2016). Esto se observa de manera acentuada entre el colectivo de estudiantes con discapacidad al atender a los factores primero, segundo y tercero, relativos a la Autoestima/Autoeficacia, a la Impotencia y a la Autonomía.

En esta misma dirección van los resultados al examinar el rendimiento académico en función de si se tiene discapacidad o no. Se observa que las puntuaciones medias 
en rendimiento académico de los estudiantes con discapacidad son inferiores a las de sus iguales sin discapacidad. En este sentido, González y Roses (2016) plantean que el empoderamiento es necesario para la obtención de resultados exitosos, y que este se define en base a cuatro características esenciales: autonomía, autorregulación, creencias de control y eficacia, y autoconocimiento. De este modo, si este colectivo muestra menos puntuaciones en estas características, es lógico que esto repercuta en los diferentes ámbitos de la vida, y más en concreto, en el contexto académico, reflejándose, por tanto, en un menor rendimiento que el obtenido por los estudiantes sin discapacidad.

Al profundizar en las otras variables del perfil sociodemográfico que puedan influir en un mayor o menor grado de empoderamiento, así como en el rendimiento académico, esto es, en las variables edad y sexo, los resultados indican lo siguiente:

En lo referente a los resultados de la edad, como factor que pueda influir en un mayor o menor grado de empoderamiento, las puntuaciones medias de esta capacidad fueron superiores en los participantes de más edad. En este sentido, a mayor edad de los participantes, mayor es la evolución en su paso por secundaria y, con ello, más posibilidades de aumentar sus conocimientos sobre diferentes situaciones, en la toma de decisiones y, en general, en mayores oportunidades para desenvolverse de manera más exitosa en una heterogeneidad de contextos. De esto se desprende que este concepto no es algo estático que sea inherente a la persona, es decir, no es un rasgo que la persona tiene o no tiene, sino que puede evolucionar a lo largo del tiempo y de las circunstancias (De los Ríos Gutiérrez y González, 2016; González y Roses, 2016; Suriá, 2018).

Estos resultados cobran mayor respaldo al atender al rendimiento académico según la edad, observándose mayor éxito académico entre el grupo de más edad. En línea con los resultados obtenidos por otros estudios (Cabanach, Farińa, Freire, González, y Del Mar Ferradás, 2013; Ramos, 2017), la dificultad de adaptación en la etapa de secundaria va a tornarse cada vez más compleja en los estudiantes más jóvenes frente al alumnado que se encuentra finalizando su paso por esta etapa. Esto se incrementa más en los estudiantes con discapacidad (Alcantud et al., 2000; Rodríguez-Martín y Álvarez-Arregui, 2015; Suriá, 2016). Una explicación plausible podría apoyarse, en parte, por la inexperiencia derivada de los propios estudiantes ante la entrada en ciclos más avanzados, sin duda, una etapa más independiente; esto unido al incremento en el nivel de dificultad de los nuevos contenidos de estudio impartidos, que pueden repercutir en que los alumnos se encuentren más desorientados y descontentos en este ciclo formativo que cuando estudiaban en primaria (Cabanach et al., 2013; Suriá et al., 2017).

En lo referente al empoderamiento en función del sexo, se advirtió que los varones mostraron un promedio superior a las mujeres en el factor relativo a la Autoestima y en el factor referente a la Autonomía. Por desgracia, la sociedad actual marca unas modas que alejan mucho a las personas con discapacidad de los cánones de la belleza (Matalinares, et al., 2013; Suriá, 2018). Esto podría repercutir en unos niveles inferiores de autoestima en gran parte de este colectivo, principalmente en las mujeres (Polo-Sánchez y López-Justicia, 2012). Asimismo, los roles de sobreprotección de los familiares todavía pueden ser factores que influyan en una menor percepción de autonomía por parte de estas mujeres.

En relación al rendimiento académico según el sexo, no se observaron diferencias significativas en los resultados. En este sentido los resultados son contradictorios. Así, aunque coinciden con los obtenidos por otros autores (Sánchez, Marín, y López, 2011) en cuanto a que el sexo femenino presenta un rendimiento académico superior al sexo masculino, otros autores han descrito que los varones tienen un rendimiento más alto que las mujeres (Mohedano y Guadalupe, 2006; Novillo, 2018). Posiblemente, como manifiestan algunas investigaciones, las posibles diferencias en el rendimiento de varones y mujeres se deben a otros elementos, tales como las distintas pautas de socialización y el refuerzo de aptitudes diferenciales por sexo (Maso, Bernet, Morales, y Úcar, 2017; Mohedano y Guadalupe, 2006; Oliva, 2006).

Con respecto al tercer objetivo, analizar la posible asociación entre empoderamiento, rendimiento académico y la variable tener o no discapacidad, los resultados revelan una relación positiva entre el empoderamiento y el rendimiento académico, y entre este y los jóvenes estudiantes sin discapacidad. Por tanto, es precisamente en el colectivo de la discapacidad donde el desarrollo del empoderamiento es prioritario, no únicamente como elemento de superación de los logros académicos, sino también como constructo en el que hay que profundizar con el objetivo de que en cualquier programa de intervención se fomente la construcción y desarrollo de esta capacidad entre las personas que conviven con esta situación. A su vez, sería conveniente seguir indagando, no únicamente en trabajos centrados en potenciar el rendimiento académico de forma directa, sino en aspectos que ayuden a fomentar otros constructos que influyan en el logro académico y, a la vez, mejoren el desarrollo personal y social de los jóvenes con discapacidad.

Este trabajo presenta algunas limitaciones que es necesario tener en cuenta. La principal radica en que, muy probablemente, los estudiantes que colaboraron tuvieran más capacidad de superación y estuvieran más motivados en participar en comparación con los que eran reacios a participar. Este aspecto sobreestimaría el grado de empoderamiento y podría sesgar la magnitud de algunas de las asociaciones detectadas. En futuras investigaciones se debería controlar este sesgo con objeto de incrementar la validez interna de los resultados. Asimismo, la muestra de participantes con discapacidad es reducida. En un futuro se espera ampliar el número de estudiantes participantes 
con este hándicap. Tampoco hay que olvidar que las particularidades experimentadas por aquellos que tienen una discapacidad son únicas para cada persona y van a estar conformadas por una compleja combinación de factores (diferencias personales de experiencias, temperamentos y contextos diferentes), que deberían haberse tenido en cuenta a la hora de explicar la variabilidad en el empoderamiento.

A pesar de estas limitaciones, y dado que los resultados reflejan que el empoderamiento se relaciona con el rendimiento académico, y que varía en función de características, como la experiencia de vivir con discapacidad, sería conveniente seguir indagando en estudios que exploren estos aspectos para permitir la mejora del diseño de programas y planes de intervención-acción, que fomenten los factores que promuevan el empoderamiento y el rendimiento académico de los jóvenes con discapacidad que se encuentran estudiando.

\section{Referencias}

Alcantud, F., Ávila Clemente, V., y Asensi Borrás, M. C. (2000). La integración de estudiantes con discapacidad en los estudios superiores. Valencia: Paidós.

Allen, J. D. (2005). Grades as Valid Measures of Academic Achievement of Classroom Learning. The Clearing House: A Journal of Educational Strategies, Issues and Ideas, 78(5), 218-223.

Barnes, C., Mercer, G., y Shakespeare, T. (1997). Exploring Disability. A Sociological Introduction. Cambridge, United Kingdom: Polity Press.

Botero, P. (2013). Discapacidad y estilos de afrontamiento: Una revisión teórica. Revista Vanguardia Psicológica Clínica Teórica y Práctica, 3(2), 196-214.

Borland, J., y James, S. (1999) The Learning Experience of Students with Disabilities in Higher Education. Disability \& Society, 14(1), 85-101.

Box, G. E., y Cox, D. R. (1964). An Analysis of Transformations. J R Stat Soc Series B Stat Methodol. 26, 211-252.

Cabanach, R. G., Fariña, F., Freire, C., González, P., y Ferradás, M. de M. (2013). Diferencias en el afrontamiento del estrés en estudiantes universitarios hombres y mujeres. European Journal of Education and Psychology, 6(1), 19-32.

Comité Español de Representantes de Personas con Discapacidad (CERMI). (2017). Empoderamiento y defensa de derechos: Guia para la autodefensa de los derechos de las personas con discapacidad 2016. Madrid: Comité Español de Representantes de Personas con Discapacidad (CERMI). Recuperado de http://hdl.handle.net/11181/5166

Cohen, J. (1992). A Power Primer. Psychological Bulletin, $112(1), 155$.

De los Ríos Gutiérrez, I., y González, M. J. C. (2016). Empoderamiento de personas con discapacidad a través del aprendizaje colaborativo: proyecto Idipower. Revista Española de Discapacidad (REDIS), 4(2), 235-240.

Elizur, D., y Magyari, I. (1993). Gender Differences in Achievement Motive: A Facet Analysis of Hungarian Samples. The Journal of Psychology, 128(1), 63-70.
Figuera Gazo, P., y Coiduras Rodríguez, J. L. (2013). La transición a la universidad: un análisis desde la diversidad de las voces de los estudiantes. Revista de Educacion, 362, 713736.

Fullan, M., y Escudero, J. M. (2002). Los nuevos significados del cambio en la educación. Barcelona: Octaedro.

Gómez-Puerta, J. M., y Cardona, M. C. (2010). Percepciones y actitudes de los padres acerca de la discriminación de sus hijos por razón de discapacidad intelectual. Educación y Diversidad: Revista Interuniversitaria de Investigación sobre Discapacidad e Interculturalidad, 4, 73-88.

González, E., y Roses, S. (2016). ¿Barreras invisibles? Actitudes de los estudiantes universitarios ante sus compañeros con discapacidad. Revista Complutense de Educación, 27(1), 219235.

Goñi, E., Madariaga, J. M., Axpe, I., y Goñi, A. (2011). Structure of the Personal Self-Concept (PSC) Questionnaire 1. International Journal of Clinical and Health Psychology, 11(3), 509-522.

Maso, P. S., Bernet, J. T., Morales, M. J., y Úcar, X. (2017). La construcción de un modelo pedagógico del empoderamiento juvenil: Espacios, momentos y procesos. Pedagogía Social: Revista Interuniversitaria, 30, 19-34.

Matalinares, M., Arenas, C., Sotelo, L., Sotelo, N., Díaz Acosta, G., Dioses Chocano, A., Yarigaño, J., Medina, R., Pezua, M., Muratta, R., y Tipacti, R. (2013). Factores personales de resiliencia y autoconcepto en estudiantes de primaria de Lima Metropolitana. Revista de Investigación en Psicologia, 14, 187-207.

Mohedano, R., y Guadalupe, M. (2006). Salud mental vs. rendimiento académico en alumnos de educación superior. Vertientes. Revista Especializada en Ciencias de la Salud, 9, 1-2.

Moore, M., Beazley, S., y Maelzer, J. (1998). Researching Disability Issues. Buckingham: Open University Press.

Novillo, S. M. (2018). Educar el talento: Guia para desarrollar nuevas habilidades en jóvenes y niños. Barcelona: Editorial AMAT.

Oliva, A. (2006). Relaciones familiares y desarrollo adolescente. Anuario de Psicología, 37, 209-223.

Oliver, M. (1998). ¿Una sociología de la discapacidad o una sociología discapacitada? En L. Barton (Ed.), Discapacidad y sociedad (pp. 34-58). Madrid: Morata/Fundación Paideia.

Peralta, Y. F. S., Gandoy, F., Jara, C., y Pacenza, M. P. I. (2016). Liderazgo del docente y niveles de empoderamiento de los estudiantes en un seminario de prácticas comunitarias de una universidad pública argentina. Cuadernos de Administración, 31(54), 68-79.

Polo-Sánchez, M. T., y López-Justicia, M. D. (2012). Autoconcepto de estudiantes universitarios con discapacidad visual, auditiva y motora. Revista Latinoamericana de Psicología, 44(2), 87-98.

Ramos, I. M. (2017). El modelo de empoderamiento en el proceso de enseñanza aprendizaje en la carrera licenciatura en Enfermería. Revista Cubana De Tecnología De La Salud, 7(3), 1-5.

Rappaport, J. (1984). Studies in Empowerment: Introduction to the Issue. Prevention in Human Services, 3, 1-7.

Rodríguez-Martín, A., y Álvarez-Arregui, E. (2015). Universidad y discapacidad. Actitudes del profesorado y de estudiantes. Perfiles educativos, 37, 86-102. 
Rogers, E. S., Chamberlin, J., Ellison, M. L., y Crean, T. (1997). A Consumer-Constructed Scale to Measure Empowerment among Users of Mental Health Services. Psychiatric Service, 48(8), 1042-1047.

Sánchez, D. G., Marín, R. O., y López, Y. E. (2011). Factores que influyen en el rendimiento académico del estudiante universitario. TECNOCIENCIA Chihuahua, 5(2), 1-8.

Segado, S. (2011). Nuevas tendencias en Trabajo Social con Familias: una propuesta para la práctica desde el empowerment. Madrid: Trotta.

Sepúlveda Rueda, N. (2008). Estrategias metodológicas para la promoción de factores protectores de la salud a través de la actividad física adaptada en personas con discapacidad cognitiva entre los 15 y 24 ańos de edad. Recuperado de http:// hdl.handle.net/123456789/926

Silva, C., Kronmüller, C., Cruz, M., y Riffo, I. (2015). Empoderamiento en el movimiento estudiantil durante 2011 y 2012 en Chile. Universitas Psychologica, 14(4), 1299-1309.
Suriá, R. (2018). Inteligencia emocional y bienestar subjetivo en estudiantes universitarios con diferentes tipos y grados de discapacidad. Bordón. Revista de pedagogía, 70(1), 125-140.

Suriá, R., Villegas, E., y Rosser Limiñana, A. (2017). Empoderamiento en estudiantes universitarios con discapacidad en función de la tipología, funcionalidad y etapa en la que se adquiere la discapacidad. Revista Española de Discapacidad (REDIS), 5(1), 63-75.

Suriá, R. (2016). Relación entre autoconcepto y perfiles de resiliencia en jóvenes con discapacidad. Electronic Journal of Research in Educational Psychology, 14(3), 450-473.

Wehmeyer, M. L. (2009). Autodeterminación y la tercera generación de prácticas de inclusión. Self-Determination and Third Generation Inclusion Practices. Revista de educación, 349, 45-67.

Zimmerman, M. (2000). Empowerment Theory. En J. Rappaport y E. Seidman (Eds.), Handbook of community psychology (pp. 43-63). Nueva York, USA: Kluwer. 